\title{
EFEITO ANALGÉSICO DA MORFINA E METADONA POR VIA EPIDURAL, ASSOCIADAS À LIDOCAÍNA EM CADELAS SUBMETIDAS À OVARIOSALPINGOHISTERECTOMIA
}

\author{
(Analgesic effect of epidural administration of morphine and methadone with \\ lidocaine in bitches submitted to ovariosalpingohisterectomy)
}

\author{
Rafaella Beatriz da Silva, Taíse Fuchs, Rafaela Galante, Sabrina Marin Rodigheri ${ }^{1}$
}

${ }^{1}$ Correspondência: rafab2007@bol.com.br

\begin{abstract}
RESUMO: A ovariosalpingohisterectomia $(\mathrm{OSH})$ é um dos procedimentos cirúrgicos mais realizados em cadelas e promove sensibilidade dolorosa moderada, sendo necessária analgesia preemptiva. A técnica de anestesia epidural pode ser utilizada com o intuito de minimizar os efeitos deletérios da dor. O objetivo deste trabalho é comparar a eficácia analgésica da morfina e metadona, associadas à lidocaína pela via epidural, bem como avaliar a incidência de efeitos adversos. Foram utilizadas 10 fêmeas caninas, aleatoriamente alocadas em dois grupos. $O$ grupo 1 recebeu morfina $(0,1 \mathrm{mg} / \mathrm{kg})$ e lidocaína $(0,3 \mathrm{ml} / \mathrm{kg})$ e o grupo 2, metadona $(0,3 \mathrm{mg} / \mathrm{kg})$ e lidocaína $(0,3 \mathrm{ml} / \mathrm{kg})$. Foram avaliados escores de sedação e dor, bem como frequência cardíaca, respiratória, temperatura corporal, pressão arterial sistólica, saturação de oxi-hemoglobina e glicemia. De acordo com os resultados obtidos pode-se concluir que tanto a morfina quanto a metadona promovem boa analgesia com mínimos efeitos colaterais, embora a metadona pareça exercer maior depressão cardiorrespiratória.
\end{abstract}

Palavras-chave: anestesia regional; dor; opioides

ABSTRACT: The ovariosalpingohisterectomy $(\mathrm{OSH})$ is one of the most common surgical procedures in bitches and promotes moderate pain sensibility, preemptive analgesia being required. The epidural technique can be used with the intention of minimizing the deleterious effects of pain. The aim of this study is to compare analgesic efficacy of epidural morphine and methadone, associated to lidocaine, and evaluate the incidence of adverse effects. Ten canine females were randomly allocated into two groups. Group 1 received morphine $(0.1 \mathrm{mg} / \mathrm{kg})$ and lidocaine $(0.3 \mathrm{ml} / \mathrm{kg})$ and group 2, methadone $(0.3 \mathrm{mg} / \mathrm{kg})$ and lidocaine $(0.3 \mathrm{ml} / \mathrm{kg})$. Sedation scores and pain as well as heart rate, respiratory rate, body temperature, systolic blood pressure, oxyhemoglobin saturation and blood glucose were evaluated. According to the results, it can be concluded that both morphine and methadone promote good analgesia with minimal side effects, although methadone seems to promote greater cardiorespiratory depression.

Key Words: opioids; pain; regional anesthesia 


\section{INTRODUÇÃO}

A ovariosalpingohisterectomia (OSH) é um dos procedimentos cirúrgicos mais comumente realizados em cadelas para prevenção de afecções reprodutivas, controle populacional ou até mesmo por opção do proprietário (DINIZ et al, 2013; POHL et al, 2012; TAMANHO et a 2010).

Mesmo durante a anestesia geral este procedimento promove nocicepção, sendo necessária a analgesia preemptiva (ou preventiva) para minimizar os efeitos deletérios da dor (SILVA E COSTA, 2011).

A anestesia epidural é uma técnica anestésica regional, segmentar e temporária, em que fármacos são depositados no canal espinhal (MASSONE, 2011), dentre eles os anestésicos locais e os opioides (SANTOS et al, 2013). Os anestésicos locais em concentrações adequadas, inibem de forma reversível a condução nervosa de fibras sensoriais e motoras, por meio do bloqueio dos canais de sódio, sendo a lidocaína o fármaco mais utilizado na Medicina Veterinária (MASSONE, 2011).

O uso isolado, no entanto, de anestésicos locais via epidural geralmente não é suficiente para o alívio da dor durante a OSH (ISHY, 2001), já que a inervação do ovário (nervo genitofemoral) se origina do $3^{\circ}$ e $4^{\circ}$ nervos lombares aferentes, estruturas muitas vezes não sensibilizadas com a técnica em doses convencionais (BAYLEY et al, 1988). Dessa forma, para obtenção de analgesia, podem-se associar fármacos opioides (COUSINS, M. J.; MATHER, 1984; MARUCIO \& COTES, 2012).
Sua ação por via epidural ocorre pela presença de grande quantidade de receptores opiáceos $(\mathrm{OP} 3-\mathrm{mi}$, OP1 delta e OPI 2 - kappa) na substância gelatinosa do corno dorsal da medula (YAKSH \& RUDY, 1976; MARUCIO \& COTES, 2012),. A melhor analgesia é obtida com o uso de agonistas mi (OP3) seletivos, como a morfina, principal alcaloide derivado do ópio, sendo à base de comparação com os demais opioides (MOREIRA, 2005; MARUCIO \& COTES, 2012; AZEVEDO, 2009; RIBEIRO et al, 2002). Há diversos relatos sobre o seu uso por via epidural, porém há poucas descrições sobre 0 uso da metadona por esta via em cães (MARUCIO \& COTES, 2012).

A metadona é um opioide sintético com efeitos farmacológicos similares ao da morfina, também atuando no receptor tipo OP3 (MARUCIO \& COTES, 2012).

Sendo assim, este trabalho tem por objetivo comparar a eficácia analgésica da morfina e metadona associadas à lidocaína pela via epidural, durante período trans e pós-operatório, em cadelas submetidas à ovariosalpingohisterectomia eletiva, bem como avaliar a incidência de efeitos adversos decorrente do uso destes fármacos.

\section{MATERIAL E MÉTODOS}

Foram utilizadas dez fêmeas caninas, com idade variando de 6 meses a 9 anos e peso de 2,2 a $34,6 \mathrm{~kg}$, saudáveis, avaliadas por meio de exame físico completo e exames laboratoriais (hemograma, dosagem de creatinina, alanina-amino-transferase e glicemia), classificadas como ASA I 
(Classificação do risco anestésico, segundo a American Society of Anesthesiologists), atendidas na Unidade de Saúde Animal I (USA I) da Faculdade Evangélica do Paraná (FEPAR). Todos os animais foram submetidos a jejum alimentar de 12 horas e hídrico de 8 horas e passaram por procedimento

de ovariosalpingohisterectomia (OSH) eletiva, sob autorização prévia dos proprietários.

Antes de qualquer manipulação do paciente foram verificadas freqüência cardíaca ( $F C)$ e freqüência respiratória (FR) por meio de estetoscopia, e temperatura corporal (TC) utilizando-se termômetro digital, por via retal, O estetoscópio e termômetro também foram utilizados durante o período de recuperação pós-operatória. Durante a cirurgia, a FC, FR e temperatura foram aferidas por meio de monitor multiparamétrico. $\mathrm{O}$ monitor também permitiu a avaliação da saturação de oxi-hemoglobina $\left(\mathrm{SpO}_{2}\right)$.

A avaliação da pressão arterial sistólica (PAS) foi realizada por meio de doppler vascular,com manguito selecionado de acordo com o tamanho do animal, posicionado próximo a articulação úmero-rádio-ulnar. A mensuração de glicemia foi realizada em três momentos (basal, logo após o término da cirurgia e 5 horas após) por meio do glicosímetro.

Nenhum tipo de medicação préanestésica foi realizada para que não ocorressem interferências durante a avaliação do escore de sedação. Após exame inicial efetuou-se tricotomia da região abdominal, da região lombossacra para administração da anestesia epidural e de membro torácico para cateterização da veia cefálica. A fluidoterapia foi realizada com solução de Ringer com lactato infundida na taxa de $10 \mathrm{ml} / \mathrm{kg} / \mathrm{h}$.

A anestesia foi promovida com a administração de propofol na dose de $6 \mathrm{mg} / \mathrm{kg}$ por via intravenosa. Os pacientes foram intubados com sondas endotraqueais, com cuff, apropriadas para seu tamanho e conectadas ao circuito anestésico para administração de isoflurano em oxigênio $\left(\mathrm{O}_{2}\right)$ a $100 \%$, por meio de vaporizador universal, ajustado para manutenção do plano anestésico cirúrgico. Os animais permaneceram sob ventilação espontânea.

Transcorrido dez minutos da indução anestésica a técnica de anestesia epidural foi executada. Os animais foram posicionados em decúbito esternal com os membros pélvicos direcionados cranialmente. Após anti-sepsia da região, a punção lombossacra foi realizada com agulha hipodérmica em animais de pequeno porte e cateter em animais de médio e grande porte. Houve a confirmação do espaço epidural através do teste da gota pendente e de resistência.

Os animais foram aleatoriamente alocados em dois grupos de 5 animais cada. O grupo I recebeu por via epidural morfina na dose de $0,1 \mathrm{mg} / \mathrm{kg}$ e o grupo II recebeu metadona na dose de $0,3 \mathrm{mg} / \mathrm{kg}$. Ambos os grupos receberam associadamente por via epidural, lidocaína sem vasoconstritor até completar a dose de $0,3 \mathrm{~mL} / \mathrm{kg}$.

Cinco minutos após a anestesia epidural, os mesmos parâmetros basais foram aferidos (exceto glicemia), acrescido da mensuração da saturação 
de oxi-hemoglobina (SpO2), avaliada por meio do monitor multiparamétrico.

O procedimento cirúrgico foi realizado sempre pelo mesmo cirurgião e iniciado após 15 minutos da aplicação da anestesia epidural. A avaliação transoperatória ocorreu em mais 5 etapas sendo: incisão da pele, pinçamento dos dois pedículos ovarianos (esquerdo e depois direito), pinçamento da cérvix e final da sutura de pele. Ao término da cirurgia, uma nova avaliação foi realizada, exatamente como a basal, inclusive a glicemia.

A duração do procedimento cirúrgico e o tempo de extubação também foram registrados. $O$ escore de sedação e dor foi avaliado após 1, 2, 3, 4 e 5 horas do término do procedimento cirúrgico. No último momento, a glicemia foi novamente mensurada.

O grau de sedação foi avaliado conforme tabela descrita por Pibarot et al (1997), utilizada para cães e graduada de 1 a 5 , onde, quanto maior o escore, maior a sedação.

Tabela 1 - Escore de Sedação de Pibarot, et al (1997)

\begin{tabular}{|c|c|c|}
\hline Patânetro & Cribéno & Pomtuaça \\
\hline \multirow[t]{5}{*}{ Grau de sedsçă } & $\begin{array}{l}\text { Acerdado, se mantèm } \\
\text { em pif e carminha }\end{array}$ & 0 \\
\hline & $\begin{array}{l}\text { Se mantém om po } \\
\text { mas incoordenado }\end{array}$ & 1 \\
\hline & $\begin{array}{l}\text { Tents, mas nẩo consegue } \\
\text { manterse em pê }\end{array}$ & 2 \\
\hline & Mantam a cabeça levantada & 3 \\
\hline & $\begin{array}{l}\text { Somerete abre os ohos } \\
\text { Sem resposta. }\end{array}$ & ${ }_{5}^{4}$ \\
\hline
\end{tabular}

A dor foi avaliada por meio da Escala de dor da Universidade de Melbourne - EDUM (Tabela 2), onde parâmetros fisiológicos e comportamentais são pontuados, podendo somar de 0 a 27 pontos, sendo 0 : ausência de dor e 27: dor máxima. Caso necessário, animais com dor acima de 13 pontos receberiam analgesia suplementar com tramadol na dose de $3 \mathrm{mg} / \mathrm{kg}$ por via intravenosa.

Após a última aferição dos parâmetros, todos os animais receberam meloxicam na dose de $0,2 \mathrm{mg} / \mathrm{kg} \mathrm{SC}$.

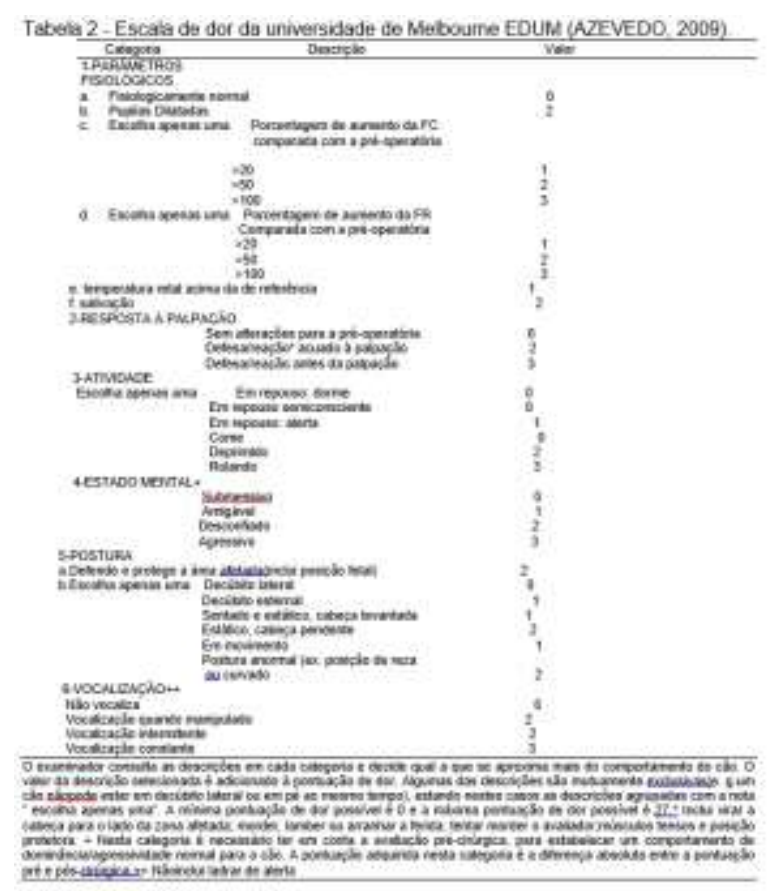

Para melhor entendimento da dinâmica do experimento, os parâmetros aferidos e os momentos de avaliação estão demonstrados no quadro 1.

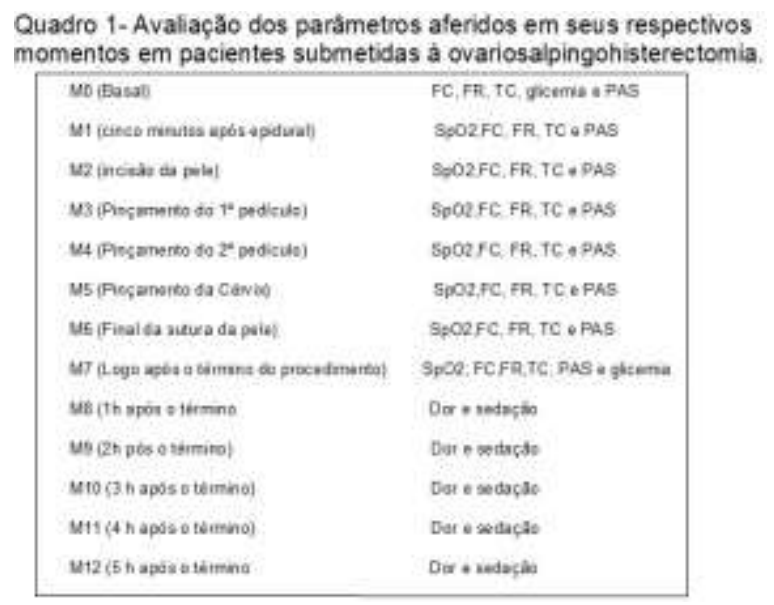




\section{RESULTADOS}

Os valores médios da frequência cardíaca $(F C)$, frequência respiratória (FR), temperatura corporal (TC), saturação de oxi-hemoglobina (SpO2) e pressão arterial sistólica durante 0 período pré e transanestésico estão listados na tabela 3.

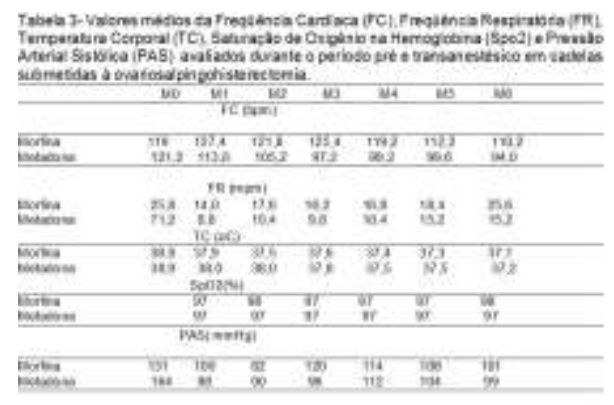

Os animais do grupo II apresentaram médias de FC mais baixas que os animais do grupo I ao longo do procedimento cirúrgico. Dois animais de cada grupo obtiveram aumento da FC cinco minutos após a epidural. Já outros dois animais de cada grupo apresentaram aumento da FC durante 0 pinçamento do $1^{\circ}$ pedículo ovariano (esquerdo), enquanto um animal de cada grupo obteve aumento da FC durante 0 pinçamento do $2^{\circ}$ pedículo ovariano (direito). Dois animais que receberam metadona e nenhum animal que recebeu morfina apresentaram aumento da FC durante o pinçamento da cérvix.

Com relação à pressão arterial sistólica (PAS) três animais do grupo I e todos do grupo II obtiveram redução desse parâmetro cinco minutos após a epidural. Os demais animais do grupo I mantiveram-se próximos aos valores basais. A redução da PAS foi mais evidente no grupo II em relação aos animais que apresentaram diminuição dessa variável no grupo I.
Em todos os animais que receberam morfina ocorreu aumento da PAS durante 0 pinçamento do $1^{\circ}$ pedículo ovariano e dois animais durante o pinçamento do $2^{\circ}$ pedículo. No grupo II ( metadona), três animais apresentaram aumento da PAS durante pinçamento do $1^{\circ}$ e do $2^{\circ}$ pedículo. Foi relatada uma maior dificuldade do cirurgião em acessar $\mathrm{o}$ pedículo ovariano nos animais que receberam a metadona pela epidural. Com relação à temperatura, todos os animais, de ambos os grupos, apresentaram redução gradativa da temperatura no trans-cirúrgico e início da recuperação anestésica.

Em relação à frequência respiratória (FR), ambos os grupos apresentaram depressão 5 minutos após a epidural (com exceção de um animal do grupo I em que a FR se manteve igual à basal neste momento); um animal de cada grupo apresentou apneia no transcirúrgico e um animal do grupo I teve aumento da FR ao final da sutura de pele. Houve melhora da depressão respiratória logo após o término do procedimento. De acordo com a média de FR, mostrada na tabela 1 , a depressão respiratória no transcirúrgico foi mais evidente com o uso da metadona do que com o uso da morfina.

Ambos os grupos obtiveram bons valores de saturação de oxigênio na hemoglobina (SpO2), com uma média de $97 \%$ em todos os momentos no grupo I e entre 97 e $98 \%$ no grupo II.

As médias de duração do procedimento cirúrgico foram 33,8 e 70 minutos para a morfina e metadona, respectivamente e a média dos tempos de extubação foram de 6 e 4 minutos, para a morfina e metadona 
respectivamente.

Com relação à glicemia (Figura 1), ambos os grupos apresentaram aumento dos valores pós-cirúrgicos em relação ao momento basal, com exceção de um animal do grupo I, que apresentou hipoglicemia.

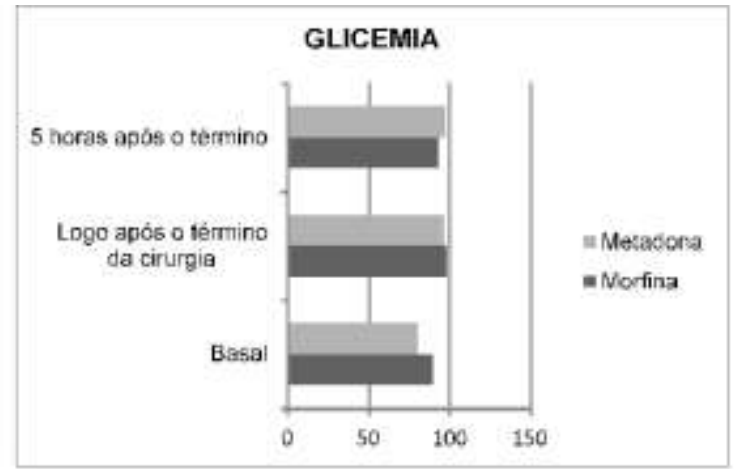

Figura 1- Mensuração da glicemia em pacientes submetidos a anestesia epidural para procedimento de ovariosalpingohisterectomia.

Todos os animais, de ambos os grupos obtiveram baixos escores de dor (Figura 2) durante a recuperação anestésica. $O$ valor máximo atingido na escala de Melbourne foi de 10 pontos, o que ocorreu em animal do G1 duas horas após o procedimento, e o valor mínimo de 1 ponto. Animais com pontuação acima de 13 pontos receberiam analgesia suplementar com tramadol, porém nenhum paciente necessitou de resgate analgésico.

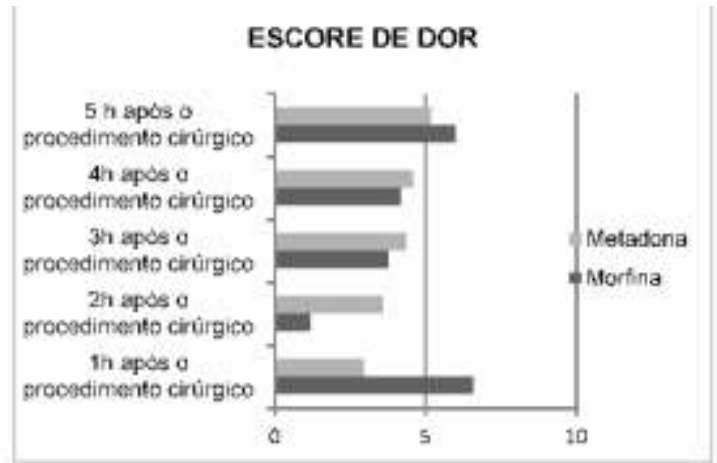

Figura 2 - Avaliação do escore de dor (Universidade de Melboume) durante o período pós anestésico em pacientes submetidos à anestesia epidural para procedimento de ovariosalpingohisterectomia.
O grau de sedação (Figura 3) diminuiu no decorrer da avaliação, sendo a média do grupo I em torno de 1 ponto e do grupo II, 3 pontos. Cinco horas após o término do procedimento cirúrgico, os pacientes apresentavam comportamento normal.

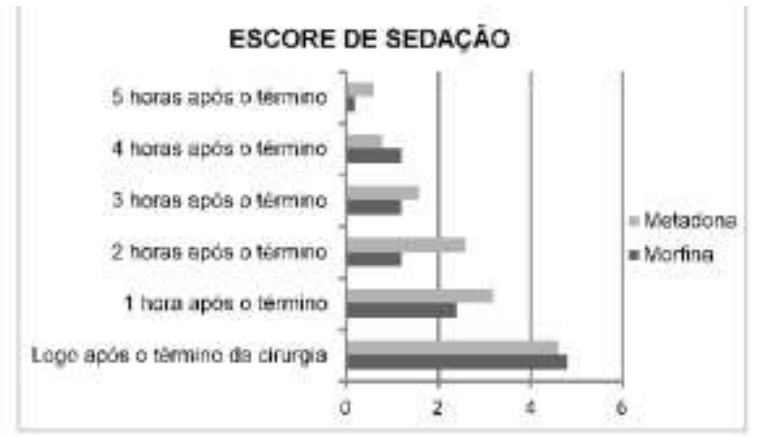

Figura 3- Avalaçăo do escore de sedaçăo (Pibarot et al. 1997) durante o periodo pós-anestésico em pacientes submetidos a anestesia epidural para procedimento de ovariosalpingohisterectomia.

Três animais do grupo I (morfina) apresentaram quadro de sialorreia na primeira hora após o procedimento, apresentando melhora em até 3 horas. Apenas um animal que recebeu metadona apresentou o mesmo quadro, porém na quinta hora. Outras alterações foram observadas no grupo da morfina ( grupo I), onde dois animais apresentaram-se ofegantes.

Não foi detectada incidência de vômito, prurido e nenhum outro efeito colateral com o uso da morfina e metadona pela via epidural.

\section{DISCUSSÃO}

A constatação de dor em animais é algo complexo, necessitando, portanto, da avaliação de diversos parâmetros fisiológicos e comportamentais relacionados à espécie observada. A administração de fármacos pela via epidural é uma das alternativas mais eficazes para 0 
tratamento da dor em animais (OTERO, 2013), sendo uma alternativa para cirurgias retroumbilicais (TORSKE \& DYSON, 2000).

Os opioides são os fármacos mais empregados na analgesia espinhal, com exceção dos anestésicos locais, principalmente devido à presença de grande quantidade de receptores opiáceos na substância gelatinosa do corno dorsal da medula (MARUCIO \& COTES, 2012; BUJEDO, et al , 2012; HAROUTIUNIAN, et al, 2013; LAURETTI et al, 2013). Em humanos, a morfina e metadona demonstraram similaridade quando aplicadas pela via epidural em diversos procedimentos (MARUCIO \& COTES, 2012).

A morfina é o protótipo dos analgésicos opioides (MOREIRA, 2005; MARUCIO \& COTES, 2012; AZEVEDO, 2009; RIBEIRO et al, 2002)., tendo grande efeito no tratamento da dor aguda e crônica. A administração epidural de um volume elevado de lidocaína associada à morfina é efetiva para realização de $\mathrm{OSH}$ em cadelas, com mínimas alterações cardiovasculares e hemogasométricas e sem depressão respiratória (TAMANHO et al, 2010).

A metadona é um opioide que atua em receptores opioides do tipo OP3 (mi), assim como a morfina, e acredita-se que possa atuar como antagonista de receptores do tipo NMDA (N-metil-D-aspartato) na medula espinhal (RIBEIRO et al, 2002, MARUCIO \& COTES, 2012, HAROUTIUNIAN et al, 2013).

Parece também bloquear a recaptação da serotonina e noradrenalina (RIBEIRO et al, 2002), o que pode ser um incremento a sua analgesia. Esse fármaco também pode prevenir o desenvolvimento de tolerância aos opioides, tendo sido usado inicialmente para prevenção da síndrome de abstinência em humanos (RIBEIRO, SCHIMIDT \& SCHIMIDT, 2002; BALTIERI et al, 2004, AZEVEDO, 2009). Apresenta alternativa efetiva no controle da dor em alguns pacientes refratários a outros opioides, principalmente no tratamento e prevenção dos portadores de dor neuropática, pelo possível papel como antagonista do receptor NMDA no sistema nervoso central (RIBEIRO et al, 2002, MARUCIO \& COTES, 2012).

Os animais que receberam metadona obtiveram médias de FC mais baixas ao longo do procedimento cirúrgico em comparação com os animais que receberam morfina. Um estudo observou bradicardia aos 15 minutos de administração tanto com metadona epidural quanto intravenosa em gatas submetidas à $\mathrm{OSH}$ (BERNARDI, 2008) em associação com a anestesia inalatória. O mesmo efeito pode ocorrer pela via intramuscular (PEREIRA, 2013, MONTEIRO et al, 2015). Há relatos de redução significativa da FC com o uso da metadona epidural e como medicação pré- anestésica, via intramuscular em cães anestesiados com isofluorano (BOSMANS, et al, 2011, MONTEIRO et al, 2015).

Porém, a redução da FC é mais evidente com a metadona associada a um anestésico local (MONTEIRO et al, 2008; BOSMANS, et al, 2011), sendo sua utilização isolada não associada à alteração cardiovascular importante (BOSMANS, et al, 2011), proporcionando estabilidade 
cardiopulmonar em cadelas submetidas à OSH (CAMPAGNOL et al, 2012; DINIZ, et al, 2013).

A bradicardia com o uso de opioides pode ocorrer devido à estimulação vagal induzida por estes fármacos e alguns animais podem apresentar pequena depressão da contratilidade miocárdica e bloqueios átrio-ventriculares de segundo ou terceiro grau (AZEVEDO, 2013), o que não foi constatado neste estudo.

Foi observada hipotensão cinco minutos após administração epidural, sendo esta mais evidente com a lidocaína associada à metadona do que com a morfina, o que também foi descrito em outros trabalhos (BERNARDI, 2008; PEREIRA, 2013). Já em outro estudo, a administração epidural de um volume elevado de lidocaína associada à morfina não resultou em diminuição adicional da PAS (TAMANHO et al, 2010). A vasodilatação, e consequente hipotensão, também podem ocorrer devido ao bloqueio simpático produzido pela anestesia epidural (BOSMANS et al, 2011).

As alterações cardiovasculares observadas podem ser explicadas pela absorção sistêmica mais rápida de opioides lipofílicos (metadona mais lipossolúvel que a morfina) no espaço epidural, causando analgesia mais rapidamente, porém com duração mais curta (PEKCAN \& KOC, 2010, CAMPAGNOL et al, 2012; BUJEDO et al, 2012; HAROUTIUNIAN, 2013).

$O$ efeito depressor cardiovascular dose-dependente de propofol em cães pode também ser um fator, principalmente naqueles animais que não receberam medicação pré- anestésica. Uma dose mais baixa produz menor depressão cardiovascular e de duração mais curta e a administração de isoflurano pode acentuar os efeitos depressores do propofol. Apesar disso, parece provável que as doses adicionais de propofol causam apenas efeitos pequenos e de curta duração (BOSMANS et al, 2011).

Outro estudo descreveu aumento gradativo da FC após administração epidural de metadona (PEKCAN \& KOC, 2010). No presente trabalho, ocorreu $o$ aumento deste parâmetro (em dois animais de cada grupo) e da PAS apenas durante 0 pinçamento dos pedículos ovarianos, provavelmente devido à tração exercida nessas estruturas durante o momento cirúrgico, - que promove intensa atividade nociceptiva (COELHO, 2013), não abolida totalmente pela técnica de anestesia regional utilizada no presente trabalho.

O tempo do procedimento cirúrgico foi maior no grupo da metadona, embora o tempo de extubação tenha sido mais curto, diferente do que observado por Pereira (2013), em que não houve diferença entre os tratamentos quanto à duração do procedimento e tempo de extubação.

Foi relatada uma maior dificuldade do cirurgião em acessar o pedículo ovariano nos animais que receberam a metadona pela via epidural, o que não foi descrito em demais trabalhos utilizando este fármaco. Dessa forma, são necessários mais estudos para comprovação deste fato.

Ambos os grupos apresentaram depressão respiratória cinco minutos após a epidural, como relatado em 
outras pesquisas (PRIETO- ALVAREZ et al., 1997;SHIR et al., 2001; DINIZ et al, 2013), que pode ser explicada pela diminuição da resposta do centro respiratório do tronco cerebral ao dióxido de carbono $\left(\mathrm{CO}_{2}\right)$ induzida pelos opioides (AZEVEDO, 2009). Dois animais do grupo I apresentaram-se ofegantes. Isso pode ser explicado devido à alteração do centro termorregulador promovida pelos opioides, o que leva a sensação de calor (AZEVEDO, 2009). Pereira (2013) não observou alterações significativas na função respiratória de cadelas submetidas à mastectomia, nem com o uso da metadona nem com o uso da morfina, tanto pela via intramuscular como pela via epidural. Tamanho et al (2009) também não constatou depressão respiratória. A depressão no trans-cirúrgico foi mais evidente com o uso da metadona se comparada à morfina.

Todos os animais apresentaram progressão da diminuição da temperatura durante o procedimento. As causas básicas da hipotermia na cirurgia decorrem de inibição central termorregulatória e da elevada perda térmica que ocasionalmente ocorrem em associação, prejudicando a termorregulação e redistribuição interna de calor, como resultado, verifica-se decréscimo na taxa metabólica. A associação de temperatura ambiente e efeito dos medicamentos préanestésicos e anestésicos (inclusive os opioides) levam a uma vasodilatação periférica, hiperventilação e a inexistência de atividade muscular agravam o quadro (MANFRINATE, 2009). Os animais estavam sob suplementação de $\mathrm{O}_{2}$ em circuito anestésico, por isso mantiveram valores adequados de saturação de oxihemoglobina.

Todos os animais de ambos os grupos obtiveram baixos escores de dor durante a recuperação anestésica e nenhum animal necessitou de resgate analgésico. Em estudo realizado, comparando-se a metadona epidural com o adesivo de fentanil, nenhum dos cães de ambos os grupos necessitaram de analgesia complementar (PEKCAN \& KOC, 2010).

Por via epidural, doses relativamente baixas de opioides produzem analgesia mais potente $\mathrm{e}$ duradoura do que a administração parenteral (ANDRADE, 2009; HAROUTIUNIAN et al, 2013). A morfina na dose de 0,1 mg/kg (OTERO, 2013) tem ação analgésica após 20 minutos de sua administração, perdurando por até 24 horas (VALADÂO et al, 2002; MARUCIO \& COTES, 2012; BUJEDO et al, 2012). Acredita-se que a metadona fique com a mesma concentração plasmática, tanto pela administração epidural quanto pela intravenosa, devido sua maior lipossolubilidade (PEKCAN \& KOC, 2010, CAMPAGNOL et al, 2012; BUJEDO et al, 2012; HAROUTIUNIAN, 2013, tendo uma duração de analgesia epidural de 4 a 6 horas (HAROUTIUNIAN, 2013), porém são necessários mais estudos para comprovação.

Nos dois grupos, a média de escore de dor reduziu gradativamente nas primeiras 3 horas, com aumento após a quarta hora, sendo este aumento mais evidente com o uso da morfina. A metadona mostrou-se viável para modulação de dor em comparação com a via intramuscular em gatas 
submetidas à OSH (BERNARDI, 2008), assim como diminuiu a nocicepção no presente trabalho, porém mais estudos são necessários para determinar a duração de ação desse fármaco por via epidural.

Com relação à glicemia, em ambos os grupos ocorreu o aumento dos valores logo após o término da cirurgia em relação ao valor basal. As mensurações

bioquímicas, especialmente o cortisol e a glicose tem se mostrado importantes referências para a avaliação da dor e do estresse por meio indireto (STURION \& SCHOSSLER, 2011).

O aumento gradativo da glicemia, logo após o procedimento e a manutenção cinco horas após indica estresse em ambos os momentos. Mesmo durante a anestesia a nocicepção se manifesta desencadeando reação de estresse de proteção do organismo animal, assim o estímulo doloroso é conduzido, chegando até 0 hipotálamo, que desencadeia resposta através do eixo hipotálamo-hipófise-adrenais, o que resulta na produção de cortisol e consequente aumento da glicemia e mesmo o uso dos analgésicos não foi capaz de impedir a resposta do organismo à manipulação dos tecidos e no pós-cirúrgico imediato, quando animais já não estavam anestesiados (SILVA \& COSTA, 2011). Além disso, fármaco anti-inflamatório

foi administrado somente depois da última aferição, o que indica que o aumento da glicemia pode estar relacionado à reação inflamatória promovida pela lesão, principalmente dos pedículos ovarianos, e liberação de várias substâncias algogênicas, que sensibilizam os nociceptores (BASSO et al, 2008).

Um animal do grupo 1 apresentou hipoglicemia gradativa ao invés de hiperglicemia. Essa redução pode ser ocorrida devido à idade do animal (6 meses). Pacientes muito jovens tem maior risco de desenvolver hipoglicemia por possuírem menor reserva de glicogênio hepático e muscular, bem como menor glicogenólise e gliconeogênese. Esses animais podem ficar em jejum por no máximo 4 horas sem desenvolver quadro de hipoglicemia (ZAGO, 2013).

Tanto a metadona quanto a morfina promoveram sedação de discreta a moderada por até $2 \mathrm{~h}$ em pósoperatório de mastectomia (PEREIRA, 2013), assim como neste estudo, onde a sedação diminuiu gradativamente no decorrer das avaliações.

A administração epidural isolada de morfina tem poucos efeitos colaterais, como retenção urinária, prurido no local da administração, constipação, vômito e hipotermia (HAYNES et al, 1993; MOREIRA, 2005). A constipação pode ocorrer com o uso dos opioides, sendo mais comum com a utilização crônica desses fármacos, já que levam ao aumento do tônus dos esfíncteres gastrointestinais e diminuição do peristaltismo. Retenção urinária é um efeito bastante comum após a administração epidural e deve-se ao fato de um aumento do tônus do músculo detrusor. Vômito e defecação são relativamente frequentes especialmente se 0 paciente não apresenta dor. A náusea e o vômito são causados pela estimulação dos quimiorreceptores existentes na zona de disparo no bulbo raquidiano, e a 
defecação resulta do aumento do tônus intestinal (AZEVEDO, 2009).

Três animais que receberam morfina apresentaram sialorreia já na primeira hora após o procedimento, o que pode ser um indicativo de náusea. Este quadro obteve melhora após 3 horas do procedimento cirúrgico. Apenas um animal do grupo da metadona apresentou salivação na quinta hora. Náusea com metadona foi observada em outros trabalhos (HAYNES et al, 1993, PEKCAN \& KOC, 2010). Entretanto, comparado aos outros opioides agonistas, a metadona apresenta menor probabilidade de induzir o vômito em cães (AZEVEDO, 2009). Não houve incidência de vômito, prurido e nenhum outro efeito colateral com o uso da morfina e nem metadona pela via epidural, condizente com resultado de outro estudo (PEKCAN \& KOC, 2010).

\section{CONCLUSÃO}

É fato que a dor promove diversas alterações importantes, o que pode ser extremamente deletério para o organismo animal. Dessa forma, o uso de analgésicos e/ou técnicas de anestesia local são necessárias para redução desses efeitos. De acordo com os resultados obtidos neste trabalho, pode-se concluir que tanto a morfina quanto a metadona associadas à lidocaína por via epidural, nas doses empregadas, promovem boa analgesia com mínimos efeitos colaterais em pacientes submetidos à OSH, embora a metadona pareça exercer maior depressão cardiorrespiratória.

\section{AGRADECIMENTOS}

A minha família pelo apoio, a Dra. Karyna Hartmman e toda a equipe da Unidade de Saúde Animal 1 (USA 1) da Faculdade Evangélica do Paraná (FEPAR), por possibilitarem a realização deste trabalho.

\section{NOTAS INFORMATIVAS}

O presente projeto foi previamente aprovado pelo comitê de ética, sob o protocolo nำ11795/ 2013.

\section{REFERÊNCIAS}

ANDRADE G. R. Anestesia epidural em cães: Revisão de Literatura. 2009. Salvador,38f. Monografia (Especialização em Clínica Médica de Pequenos Animais) - Universidade Federal Rural do Semi-Árido.

AZEVEDO P. R. A. S. Problemática da dor no período peri-operatório em canídeos. $2009 . \quad$ Lisboa,154f. Dissertação (Mestrado Integrado) Faculdade de Medicina Veterinária, Universidade Técnica de Lisboa,

BAYLEY C. S.; KITCHEL R. L.; HAGHIGHI S. S. Spinal nerve root origins of the cutaneous nerves of the canine pelvic limb. American Journal of Veterinary Research, v. 49, n. 1, Davis, 1988.

BASSO P. C.; RAISER A. G.; CARREGARO A. B. MÜLLER D. C. M. Analgesia transoperatória em cães e gatos. Revista Clínica Veterinária. Ano 13, n. 77, p. 62- 67, São Paulo, 2008.

BERNARDI C.A.; CASSU R. N.; BALAN, J. O. et al. Comparação do efeito analgésico da metadona via intramuscular ou epidural em gatas submetidas à ovariosalpingohisterectomia. ANAIS Orais pesquisa 2008. Presidente Prudente, Universidade do Oeste Paulista. 
BOSMANS T.; SCHAUVLIEGE S.; GASTHUYS F. et al. Cardiovascular effects of epidural administration of methadone, ropivacaine $0.75 \%$ and their combination in isoflurane anaesthetized dogs. Veterinary Anaesthesia and Analgesia, v. 38, n. 2, p. 146-57, Merelbeke, 2011.

BUJEDO, B.M.; SANTOS, S.G.; AZIPIAZU, A.U. A review of epidural and intrathecal opioids used in the management of postoperative pain. Journal of Opioid Management . v. 8 n. 3 pg 177 192, 2012.

CAMPAGNOL, D.; TEIXEIRA-NETO, F. J.; PECCININI, R. G et al. Comparison of the effects of epidural or intravenous methadone on the minimum alveolar concentration of isoflurane in dogs. The Veterinary Journal, v. 192, n. 3, p. 311315, 2012.

COELHO K. Efeitos da morfina intramuscular sobre a concentração alveolar mínima do isofluorano, a resposta neuroendócrina no período trans-operatório e a dor pósoperatória em cadelas submetidas à ovariosalpingohisterectomia. Vila Velha, 56f. Dissertação (Mestrado em Ciência Animal) - Universidade Vila Velha - ES.

DINIZ M. S.; KANASHIRO G. P.; BERNARDI C. A. et al. Extradural anesthesia with lidocaine combined with fentanyl or methadone to ovariohisterectomy in dogs. Acta Cirúrgica Brasileira, v. 28, n. 7, p 5316, São Paulo, 2013.

HASKINS S. C.; FARVER T. B.; PATZ J. D. Cardiovascular changes in dogs given diazepam and diazepamketamine. American Journal of Veterinary Research, v. 47, n. 4, p. 795-8, 1986.

HAROUTIUNIAN, S.; KAGAN, L.; YAFRACH-DAMARI, l. et al. Enhanced antinociceptive efficacy of epidural compared with i.v. methadone in a rat model opf thermal nociception. British Journal of Anaesthesia pg 1-9. 2013.

ISHY H. M. Uso da lidocaína isolada ou asssociada à quetamina ou ao butorfanol em anestesia epidural em cães: avaliação cardiorrespiratória e analgésica. 2001. Botucatu, $95 f$. Dissertação (Mestrado em Anestesiologia) - Universidade Estadual Paulista "Júlio de Mesquita Filho" Faculdade de Medicina.

KLAUMANN P. R.; WOUK A. F. P. F.; SILLAS T. Patofisiologia da Dor (Pathophysiology of pain). Archives of Veterinary Science, v. 13, n. 1, p. 1-12, Curitiba, 2008.

LAURETTI, G.R.; RIZZO, C. C.; MATTOS, A. L. et al. Epidural methadone results in dose-dependent analgesia oin cancer pain, further enhanced by epidural dexamethasone. British Journal of Cancer, 2013, 108; 259-264.

MANFRINATE R.; DAHROUG M. A. A.; FARIAS D. C. et al. Efeitos da morfina e da metadona associadas à acepromazina em gatas anestesiadas com propofol e halotano e submetidas à ovariosalpingohisterectomia.

Acta Scientiae Veterinariae, v. 37, n. 3, p. 245-251, Porto Alegre, 2009.

MARUCIO R.; COTES L. Fármacos de uso espinhal. In: FANTONI D. Tratamento da Dor Clínica de pequenos Animais, Rio de Janeiro Elsevier, 2012.

MASSONE F. Anestesia Local. In: MASSONE F. Anestesiologia Veterinária, farmacologia e técnicas: texto e atlas colorido. 6 ed., Rio de janeiro, Guanabara Koogan, 2011.

MATHEWS K. A. Dor: origem e efeito. In: RABELO R. D.; CROWE J. R. D. Fundamentos de terapia intensiva veterinária em pequenos animais: condutas no paciente crítico, Rio de janeiro, L F. Livros, 2005. Cap. p. 519- 
527.

MONTEIRO, E. R.; COELHO K.; BRESSAN $T$, et al. Effects of acepromazine- morphine and acepromazine- methadone premedication on the minimum alveolar concentration of isoflurane in dogs, Veterinary Anaesthesia and Analgesia, 2015.

MOREIRA J. C.. Controle da dor em UTI: identificação da dor através do comportamento. In: RABELO R. D.; CROWE J. R. D. Fundamentos de terapia intensiva veterinária em pequenos animais: condutas no paciente crítico, Rio de janeiro, L F. Livros, 2005. Cap. P. 722- p.

OTERO P. E. Anestesia locorregional do neuroeixo. In: KLAUMANN P. R.; OTERO P. E. Anestesia locorregional em pequenos animais, São Paulo, Roca, 2013.

PECKAN Z. KOC B. The post-operative analgesic effects of epidurally administered morphine and transdermal fentanyl patch after ovariohysterectomy in dogs. Veterinary Anaesthesia and Analgesia. v. 37, n. 6, 2010. Cap. p.557-565.

PEREIRA V. G. Efeito analgésico da metadona e morfina intramuscular ou epidural, associadas ou não à lidocaína, em cadelas submetidas à mastectomia. 2013. Viçosa, 44f. Dissertação (Pós-graduação em Medicina Veterinária) - Universidade Federal de Viçosa.
PIBAROT P.; DUPUIS J. GRISNEAUX E. et al. Comparison of ketoprofen, oxymorphone hydrochloride, and butorphanol in the treatment of postoperative pain in dogs. Journal of the American Veterinary Medical Association, v.211, n. 4, p 438-44, 1997.

POHL V. H.; CARREGARO A. B.; LOPES C.et al. Epidural anesthesia and postoperatory analgesia with alpha-2 adrenergic agonists and, lidocaine for ovariohysterectomy in bitches. Canadian Journal of Veterinary Research. v. 76, n. 3, p. 215-20, Ottawa, 2012.

RIBEIRO S.; SCHMIDT A. P.; SCHMIDT S. R. G. O. O uso de opioides no tratamento da dor crônica não oncológica: o papel da metadona Revisão. Revista Brasileira de Anestesiologia, v. 52, n. 5, p. 644-651, Rio de Janeiro, 2002.

SANTOS G. J.; PIRAJÁ G. V.; DIAS L. G. G. G.; PEREIRA D. M. Anestesia epidural em pequenos animais Revisão. Revista Científica Eletrônica de Medicina Veterinária [periódico online], v. 7, n. 2, Garça, 2009. Disponível

em:<http://www.revista.inf.br/veterinaria 12/revisao/pdf/AnoVIIEdic12Rev07.pdf>. Acesso em: 02/08/2013.

SILVA F. L.; COSTA A. P. R. Analgesia preemptiva em cadelas submetidas à ovariosalpingohisterectomia: avaliação dos parâmetros fisiológicos e bioquímicos. Pesquisa Veterinária Brasileira.n.0 v.0 p 0-0. Universidade federal do Piauí, Teresina, 2011.

STURION A. L. T.; SCHOSSLER J. E. D. Cortisol, glicose e triglicerídeos na resposta neuroendócrina de cães com fratura tibial. 2011. Santa Maria, 37f. Dissertação (Mestrado em Cirurgia Veterinária) - Universidade Federal de Santa Maria.

TAMANHO R. B.; OLESKOVICZ N.; MORAES A. N.et al. Anestesia epidural cranial com lidocaína e morfina para 
campanhas de castração em cães. Ciência Rural, v.40, n. 1, Santa Maria, 2010.

TORSKE K. E.; DYSON D. H. Epidural analgesia and anesthesia- Review. Veterinary Clinics of North America: Small Animal Practice, v. 30, n. 4, p. 859-74, 2000.

ZACLIKEVIS M. V.; D'AGULHAM A. C.; BERTASSONI L. E. et al. Effects of benzodiazepine and pilocarpine on rat parotid glands: histomorphometric and sialometric study. Journal of Medicinal Chemistry, v. 5, n. 1, p. 74-8, Washington D.C, 2009.

ZAGO B. S. Prós e contras da castração precoce em pequenos animais. 2013. Porto Alegre, 30f. Monografia (Graduação em Medicina Veterinária) - Universidade Federal do Rio Grande do Sul.

MONTEIRO, E. R.; DOSSI, R. J. O.; ANTUNES A. P. et al. Efeitos da metadona ou do neostigmine, assiciados à lidocaína administrados pela via epidural em cães. Arquivo Brasileiro de Medicina Veterinária e Zootecnia. V.60, .6, p.1239-1446, Botucatu, 2008.

YAKSH, T. L.; RUDY, T. A. Analgesia mediated by a direct spinal action of narcotics. Science, v. 192, n. 4246, p. 1357-1358, 1976.

BALTIERI, D. A.; STRAIN, E. C.; DIAS, J. C. et al. Diretrizes para o tratamento de pacientes com síndrome de dependência de opioides no Brasil. Revista Brasileira de Psiquiatria, v. 26, n. 4, p. 259-269, 2004.

HAYNES, S.R.; DAVIDSON, I.; ALLSOP, J.R. et al. Comparison of epidural methadone with epidural diamorphine for analgesia following caesarean section. Acta Anaesthesiologica Scandinavica, v.37, p.375-380, 1993.
COUSINS, M. J.; MATHER, L. E. Intrathecal and epidural administration of opioids. Anesthesiology, v. 61, n. 3, p. 276-310, 1984.

PRIETO-ALVAREZ,

M.P.; FUENTESBELLIDO, J.G.; LOPEZCEBOLLADA, J. et al. Comparative study of postoperative analgesia with methadone and fentanyl in continuous peridural perfusion. Revista Española de Anestesiología y Reanimación, v.44, p.305-309, 1997.

SHIR, Y.; ROSEN, G.; ZELDIN, A. et al. Methadone is safe for treating hospitalized patients with severe pain. Canadian Journal of Anesthesia, v.48, p.1109-1113, 2001. 